\title{
COVID-19 Outbreaks and Age Mortality Patterns
}

Vladimir Shapiro ( $\nabla$ v.shapiro@northeastern.edu )

Northeastern University, Boston, MA, United States https://orcid.org/0000-0001-7397-3637

\section{Research Article}

Keywords: COVID-19 outbreak, COVID-19 wave, age mortality gradients, outbreak age vulnerability, death aging

Posted Date: November 24th, 2020

DOI: https://doi.org/10.21203/rs.3.rs-113791/v1

License: (c) (1) This work is licensed under a Creative Commons Attribution 4.0 International License. Read Full License 


\section{Abstract}

As of the Fall of 2020, many countries are still fighting the COVID-19 pandemic. After the painful first massive wave in the Spring, more and more of them are facing the new outbreaks of varying impact. Understanding the mortality pattern associated with such subsequent outbreaks would help governments better prepare and save lives. These secondary outbreaks are still quite new to the scientists as the data have not been sufficient to identify robust trends. By now, US is dealing with the second outbreak of large magnitude and statistically significant analyses are finally possible.

We have analyzed the weekly mortality death counts for various ages in US for the entire COVID-19 pandemic duration. Three somewhat related features involving age at death have been extracted: a) COVID-19 average age at death, b) fraction of deaths at ages 65+, and c) slope of age gradient regression line on the logarithmic scale. It turns out that during the outbreak the mortality age gradient is undergoing the following changes: a) average age at death at the peak is $4-5$ years higher than at the lower point; b) fraction of deaths of $65+$ is by approximately $10 \%$ higher, and c) the higher slope of the age gradient translates into an extra death risk of $5.8 \%$ every year. In other words, risks, to which an elderly population is exposed during all phases of the pandemic, rise sharply during and right after the outbreak peaks.

\section{Introduction}

As the world grapples with COVID-19 pandemic havoc, scientists have been busy with analyzing the data and uncovering underlying trends. The stakes are high: loss of human lives and devastating costs to the economies should be minimized. The first wave/outbreak of the pandemic seems to have subsided in most of Europe, East Asia, Oceania, Middle East. There are, however, early signs of COVID-19 resurgence in Israel, Spain, France, UK, Russian Federation, Australia and other countries, where local restrictions or even full-fledged lockdowns are being re-introduced. The absolute death counts are still low in these countries to draw meaningful conclusions. United States stands out in comparison: it has experienced the first outbreak which started in March of 2020 with $120,000+$ deaths accumulated by mid-June when it subsided to the levels 4-5 times lower. Since, the death counts began growing again in July-August, as seen on Figure 1. A word on the terminology: the literature is ambiguous about how to refer to the latter death count surge: some consider it being a second wave (Hasan, 2020), others argue that the first wave had not really ended and speak about second "peak" e.g. (Lopez et al, 2020), "outbreak", "outburst" or "spike" (Maragakis, 2020). We will use the less controversial term "outbreak" below.

Little is known about the mortality structure of the second or subsequent outbreaks. Among the countries experiencing it the numbers of perished have been insignificant so far: in some cases, e.g. Israel, where the outbreak had started earlier, it is due to a smaller population of the country being just under 9 million. In other, larger countries, e.g. France or Spain, the low numbers of victims are due to the much later start of the recent outbreak. In this context, however, US turned out to be in a unique position: country's population of nearly 330 million people and two months into the second outbreak as of time of writing (September 2020), are the reasons for the relatively large mortality numbers. This paper studies the US official COVID-19 data aiming at identifying trends that would advise other countries, states or territories in what they should expect if and when the COVID-19 pandemic strikes there again.

Looking at COVID-19 death rate time series in the US, we can observe one fully shaped mode with a clear rising and falling fronts in the period between late March and June 2020. The second, lower mode, had emerged in July, peaked in early August and has been gradually declining since, see Figure 1. Neither of the two modes has ever subsided to zero. Yet, we hope that the two well developed modes allow drawing meaningful conclusions.

COVID-19 mortality statistics have been widely researched in e.g. Demombynes (2020), Sobotka et al (2020), Guilmoto (2020), Goldstein (2020), supporting the following main age-related trends:

- Older populations are at higher death risks.

- Mortality age gradients are shaped exponentially following the Gompertz's law.

The COVID-19 and the general mortality data used in the research originate from the weekly mortality datasets from US government (National Center for Health Statistics CDC , 2020[2]) for the entire duration of the pandemic from the early days in March 2020 until now (September 2020).

[2] CDC disclaimer warns that number of deaths reported in this table are reported and entered with a delay which can range from 1 week to 8 weeks or more. This may affect the very recent weeks in this paper.

\section{Materials And Methods}

Our working hypothesis is that various phases of COVID-19 outbreak would affect the shape of the age mortality gradients. We will use the first outbreak (March - June) for spotting trends, then we will see if these trends persist in the mortality data from the second outbreak.

COVID-19 does not kill by itself, rather it plays a role of a trigger of other conditions and deceases. As stated in (Goldstein, 2020), with respect to the age gradients, "increase in mortality by age from COVID-19 strongly resembles the age pattern of all-cause mortality". The latter definitely holds true when the COVID-19 mortality data is analyzed all at once. In this paper, however, we are placing the mortality data on the time axis and analyzing the weekly dynamic of age gradients. Further, we postulate here that when the levels of COVID-19 activity, and, consequently, the death rates are low, the age gradients tend to remind on the non-COVID-19 ones. This is the situation between the outbreaks. However, when the outbreak occurs and death rates accelerate, the age gradients would deviate from the pre-outbreak levels. This can be observed on Figure 2. At this point we do not have a solid theory for this artifact genesis. One possible explanation can be in the spillover from the younger, less vulnerable but more exposed to the virus, population, to the older one, which is more vulnerable but is less exposed under no-outbreak circumstances. Thus, we would expect the lower fraction of deaths among the vulnerable populations before and after the outbreak, and higher during the active outbreak. 
We dissected the age mortality for the duration of an outbreak with the following three features that are closely related, but allowing slightly different interpretations:

- Average Age at COVID-19 Death

- Fraction of Age of 65+ Deaths from Total COVID-19 Death Count.

- Slope of the fitted regression line to the standardized mortality age gradients in the logarithmic scale

\section{Results}

\subsection{Average Age at COVID-19 Death}

Death rate in time for each outbreak distribution has a shape reminiscent on a left-skewed bell-curve, see Figure 1. Shape of the average death age distribution is somewhat similar with the flatter top and slightly longer right tail, see Figure 2 in red. The interpretation of the plot is that the average age death at a "dormant" state of COVID-19 pandemic has been about 73[3]. With the surge in death counts and upon the lag of 1-2 weeks, the average age death starts climbing up to 76-77 for both outbreaks. About 4-5 weeks after the death count reaches a peak (see Figure 2 in black), the average death (see Figure 2 in red) follows suit and commences a decline to the regular pre- outbreak levels. The average age spread between the pre-outbreak and the peak is $3-4$ years. The lag of 4-5 weeks between the peaks is consistent with the range of 2-7 weeks (median of 25 days) reported by (Barman, 2020) for COVID-19 patient recovery or death.

\subsection{Fraction of Age of 65+ Deaths from Total COVID-19 Death Count}

Shape of the time series of the fraction of deaths at $65+$, see Figure 4, is very similar to Figure 3 . It is not surprising since both metrics are highly correlated; the higher the average age, the higher the fraction of $65+$ ages. Yet, the latter may be easier to interpret than the average age one which, naturally, affect all the ages. The fraction of $65+$ varies between $74 \%$ and $82 \%$ for the duration of the outbreak, which is about $10 \%$.

\subsection{Slope of The Fitted Regression Line to The Standardized Mortality Age Gradients}

This feature is a bit more difficult to obtain and interpret compared to the previous two, however it provides a deeper insight into the underlying trends. It had been established that COVID-19 mortality tends to follow Gompertz's law, increasing exponentially at a constant rate (Goldstein, 2020). Interpreting quasiexponential curves of mortality age gradients is unnatural for a human eye, see Figure 5(a), therefore it is customary to plot exponential data on a logarithmic scale as shown on Figure 5(b)[4], and fitting a simple regression log-linear model to the fatality counts in a manner similar to (Demombynes, 2020; Guilmoto, 2020):

$$
\log (y)=b_{0}+b_{1} x
$$

where $y$ represents $\mathrm{F}_{\text {age }}$ and $x$ represents an age group. here stands for Fatality Count, standardized per 100,000 of each age group in general population (United Nations - Male, 2020; United Nations - Female, 2020). Simple linear regression line is fit to obtain the age gradient slope, see the dashed lines in Figure $5(b)$. Steeper, pointing higher, regression lines indicate relatively more numerous deaths for older ages, and, consequently, higher average death age. Death is "aging" faster, so to speak. Lower slopes mean fewer deaths for elderly and lower average death age. Using the regression models from Figure 5 we can calculate that the "high slope" week increases the risk of dying by $282 \%$ with an increment 10 years of age versus $224 \%$ for the "low slope" week, i.e. an extra $58 \%$ or additional $5.8 \%$ each year.

Intepretation of the higher slope (is that the risk of dying from COVID-19 is growing faster. The time series for are shown on Figure 6 (in red) to compare with absolute death count. The pattern for the second COVID-19 outbreak matches the first for the most part; we are still awaiting the return of the slopes to low levels in the forthcoming weeks.

[3] Original data from (National Center for Health Statistics CDC, 2020) had an open interval for "85 Over" age group. In calculations we have used "90" as a representative data point for this group throughout this paper. For the rest of the closed intervals we used the middle point, e.g. "70" for the "75-84".

[4] Age groups of under 20 with nearly zero fatalities have been excluded from the analysis.

\section{Discussion}

Based on the weekly data from CDC (National Center for Health Statistics CDC, 2020) we have observed that the COVD-19 outbreak affects the age mortality in a particular way: namely by causing the uptick in average death age. The data is statistically significant and the trend has manifested itself for both outbreaks experienced in the US so far.

What is the mechanism that causes this pattern to form ? We do not have a solid theory for it and purpose of this research is to identify the pattern rather than finding behavioral or bio-medical explanations. Still, an intuition for the correlation between the COVID-19 age mortality gradient behavior in time and the outbreak phases can be as follows. We will put aside the first outbreak for now; it had taken the world by surprise overwhelming insufficiently equipped hospitals, wreaking havoc on the elderly care facilities, encountering a general population lacking safely habits and personal protection. These factors have been acknowledged since and largely taken care of. It is therefore more relevant to look forward assuming that the first outbreak lessons had been learned and the past mistakes will not be repeated. Let us then focus on the second and subsequent outbreaks which likely would be similar until effective medications and viable vaccines become widely available. 
When the first pandemic outbreak had subsided, the general risk awareness was still high while the infection spread was low. Vulnerable sub-populations of elderly were diligently protecting themselves avoiding unnecessary contacts with the outside world. Later, the more outgoing and socially active younger individuals, who belonged to the low risk groups, exhibited less restrain getting infected in growing numbers and thus causing the second outbreak. More infections among the youth led to marginally more deaths than previously; just enough to lower the death age compared to the peak which hit the elderly hardest. Infections spread further; the younger individuals, e.g. stuffers in nursing homes, got in contact with the elderly often infecting them. This triggered the process of elderly dying in larger numbers again, as in the first outbreak, manifested in the death "aging", i.e. age at death growing. When the outbreak became a public priority and more strict mitigation measures, e.g. lockdowns, were introduced, relatively fewer new vulnerable individuals got infected while the already infected ones either passed away or healed. The latter developed antibodies which temporarily excluded them from the target for the virus population. At this point, the falling front started occurring and the death age begun declining again. The outbreak was subsiding; the loop was closing. As of mid-September 2020, we are observing the outbreak already passing the death count peak but the death age is still staying high. If the pattern typical to the first outbreak is persistent, this should start happening in a few weeks. The outbreak dynamic is perhaps analogous to the forest fires that start with the peripheral grass and bushes catching fire. The "core" of the forest is still intact but the flames are gradually closing in. This is the rising front, when relatively more younger age individuals (grass and bushes) die. At some point, the infections spread to a degree when contacts with the virus cannot be avoided, and the defense lines get penetrated. The fire reaches the most vulnerable core, at this point the fire (pandemic) is left to run its natural course very much like it did during the previous outbreak exhibiting the same lethal mortality patterns.

One may argue that the US is too large and diverse and, as such, may not be representative for other countries but a very few similar size ones. Indeed, US consists of individual states which may be far apart geographically from each other, have different climate, social and demographic conditions. COVID-19 pandemic has thus been spreading with different intensity and timing. The Northeastern states, e.g. New York, New Jersey, have only seen a single outbreak in the Spring. But a number of others such as California, Texas, Florida, Illinois, Louisiana, etc. have experienced the second outbreak in late Summer of actually larger than the first one's magnitude. What is important, and what makes the US case relevant to our countries and territories, is that by the time the second outbreak stroke, there had been a general awareness of the danger and risks associated with COVID-19. It had not always been the case during the first outbreak, yet, and in spite of the mature public attitude, the better healthcare system preparedness, the age mortality patterns of both outbreaks bear obvious similarities which are statistically significant. It suggests that such patterns of COVID-19 age at death may re-emerge with each subsequent outbreak in any place on Earth.

Can we speak about a cyclical nature of the outbreaks spread by 3-4 months apart ? So far, the data on Figure 1 in the US and a number of other countries mentioned above may suggest that. It could be due to the alleged rapid decay in antibodies among the individuals recovered from COVID-19 (Ibarrondo et al, 2020) during the previous outbreak that take roughly 3-4 months to occur, among the other reasons to be looked into.

It has been long established that the risk of dying from COVID-19 grows exponentially with age (Goldstein et al, 2020). Findings of this work show the risks for the elderly during the outbreaks are substantially higher that the averages across the entire pandemic. In the two outbreaks in the US the risk increase at the outbreak peak would be up to $5.8 \%$ every year of life compared with risk between the outbreaks.

See the code repository (Shapiro, 2020) to recreate the results as of the time of writing and generate the up-to-date ones including additional plots.

\section{Conclusions}

Drawing from the weekly historical mortality data from US government CDC dataset with over 200,000 COVID-19 fatalities and $1.6 \mathrm{M}$ total deaths for the yearto-date, this research analyzed the age mortality dynamic for the period spanning the two COVID-19 outbreaks so far. Among the features considered were the COVID-19 average age at death, the fraction of deaths at ages 65+, and the slope of standardized age gradient regression line on the logarithmic scale. The data shows that during the outbreak the effect of the death "aging" is taking place. The average age at death is going up by 3-4 years at the outbreak peak; the fraction of age at death at $65+$ is growing by approximately $10 \%$; risk of dying is increasing $5.8 \%$ each year of life. Awareness of such age mortality dynamic during the COVID-19 outbreak may prevent unwarranted deaths and save lives if protective actions are taken early on.

\section{References}

Hassan, E. M. \& Mahmoud. H., (2020). Impact of COVID-19 Second Wave on Healthcare Networks in the United States, medRxiv 2020.07.11.20151217; doi: https://doi.org/10.1101/2020.07.11.20151217

López, L. \& Rodó, X. (2020). The end of social confinement and COVID-19 re-emergence risk. Nat Hum Behav, 4, (pp.746-755). https://doi.org/10.1038/s41562-020-0908-8

Maragakis, L. (2020). First and Second Waves of Coronavirus, https://www.hopkinsmedicine.org/health/conditions-and-diseases/coronavirus/first-andsecond-waves-of-coronavirus, Accessed on August 12, 2020

Demombynes, G., (2020). COVID-19 Age-Mortality Curves Are Flatter in Developing Countries. Policy Research Working Paper; No. 9313, World Bank, Washington, DC.

Sobotka, T., et al., (2020). Age, gender and COVID-19 infections, medRxiv 2020.05.24.20111765; doi: https://doi.org/10.1101/2020.05.24.20111765

Guilmoto, C., (2020). COVID-19 death rates by age and sex and the resulting mortality vulnerability of countries and regions in the world, medRxiv

2020.05.17.20097410; doi: https://doi.org/10.1101/2020.05.17.20097410. Accessed on 25 July, 2020

Page $4 / 14$ 
Goldstein, J.R. \& Lee, R.D. (2020). Demographic perspectives on the mortality of COVID-19 and other epidemics, PNAS, Aug 2020, 202006392; DOI: $10.1073 /$ pnas.2006392117

National Center for Health Statistics CDC, (2020). Provisional COVID-19 Death Counts by Sex, Age, and Week https://data.cdc.gov/NCHS/Provisional-COVID19-Death-Counts-by-Sex-Age-and-W/vsak-wrfu, Accessed on September 25, 2020

Barman, M. et al, (2020). COVID-19 pandemic and its recovery time of patients in India: A pilot study, 14, https://doi.org/10.1016/j.dsx.2020.07.004, Accessed on September 12, 2020

United Nations - World Population prospects 2019, "Quinquennial Population by Five-Year Age Groups - Male", https://population.un.org/wpp/Download/Files/1_Indicators\%20(Standard)/EXCEL_FILES/1_Population/WPP2019_POP_F07_2_POPULATION_BY_AGE_MALE Accessed 5 May 2020.

United Nations - World Population prospects 2019, "Quinquennial Population by Five-Year Age Groups - Female", https://population.un.org/wpp/Download/Files/1_Indicators\%20(Standard)/EXCEL_FILES/1_Population/WPP2019_POP_F07_3_POPULATION_BY_AGE_FEMA , Accessed 5 May 2020.

Ibarrondo, F., et al, (2020). Rapid Decay of Anti-SARS-CoV-2 Antibodies in Persons with Mild Covid-19, N Engl J Med; 383:1085-1087, DOI: 10.1056/NEJMc2025179

Shapiro, V., (2020). Supplement source code, GitHub Repository, https://github.com/vladimir-shapiro/COVID_CDC_Wave-Age-Trends

\section{Figures}

\section{Age Structure of COVID-19 Deaths}

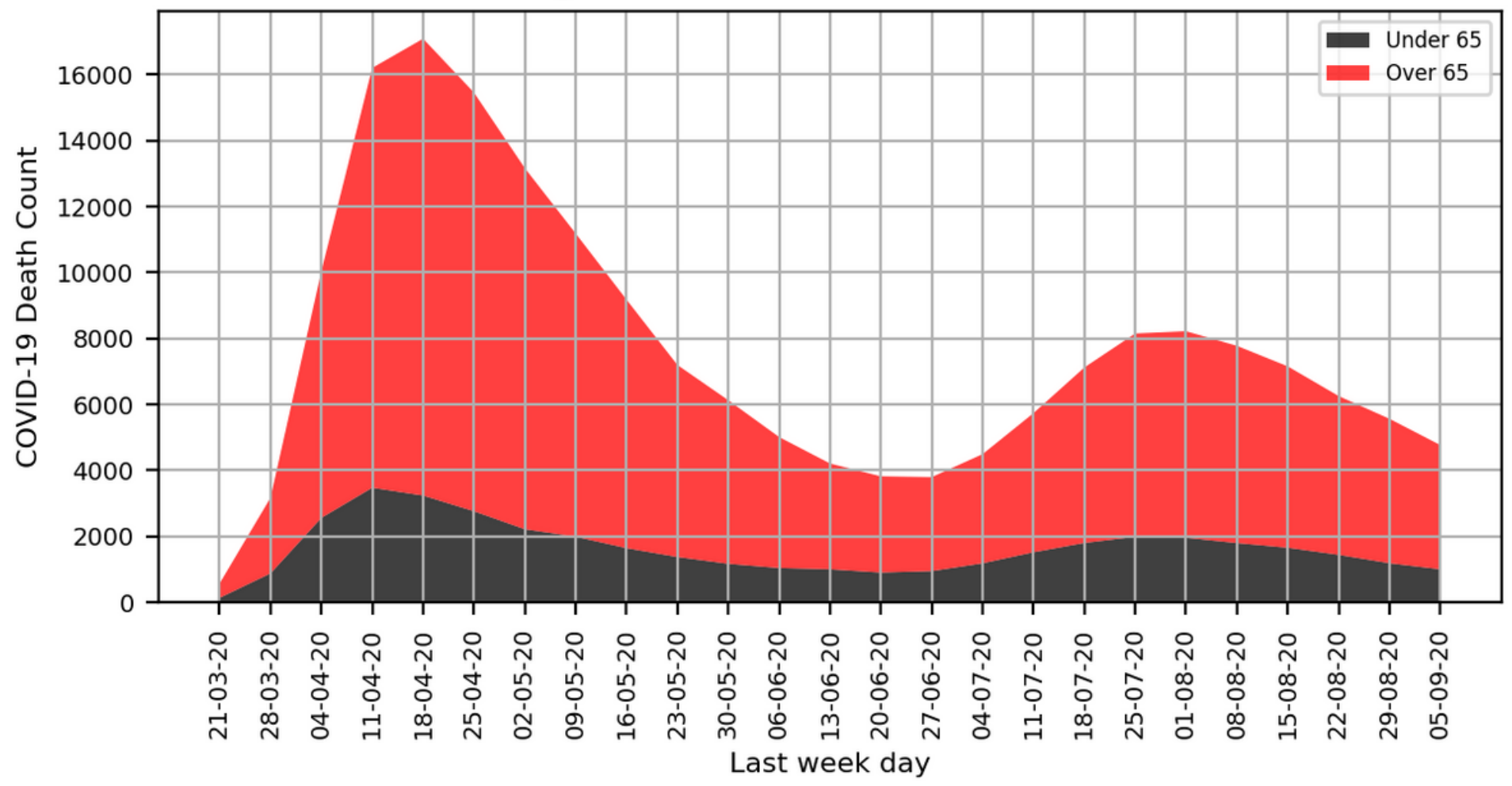

Figure 1

Weekly COVID-19 death count with age contribution. 
Age Structure of COVID-19 Deaths

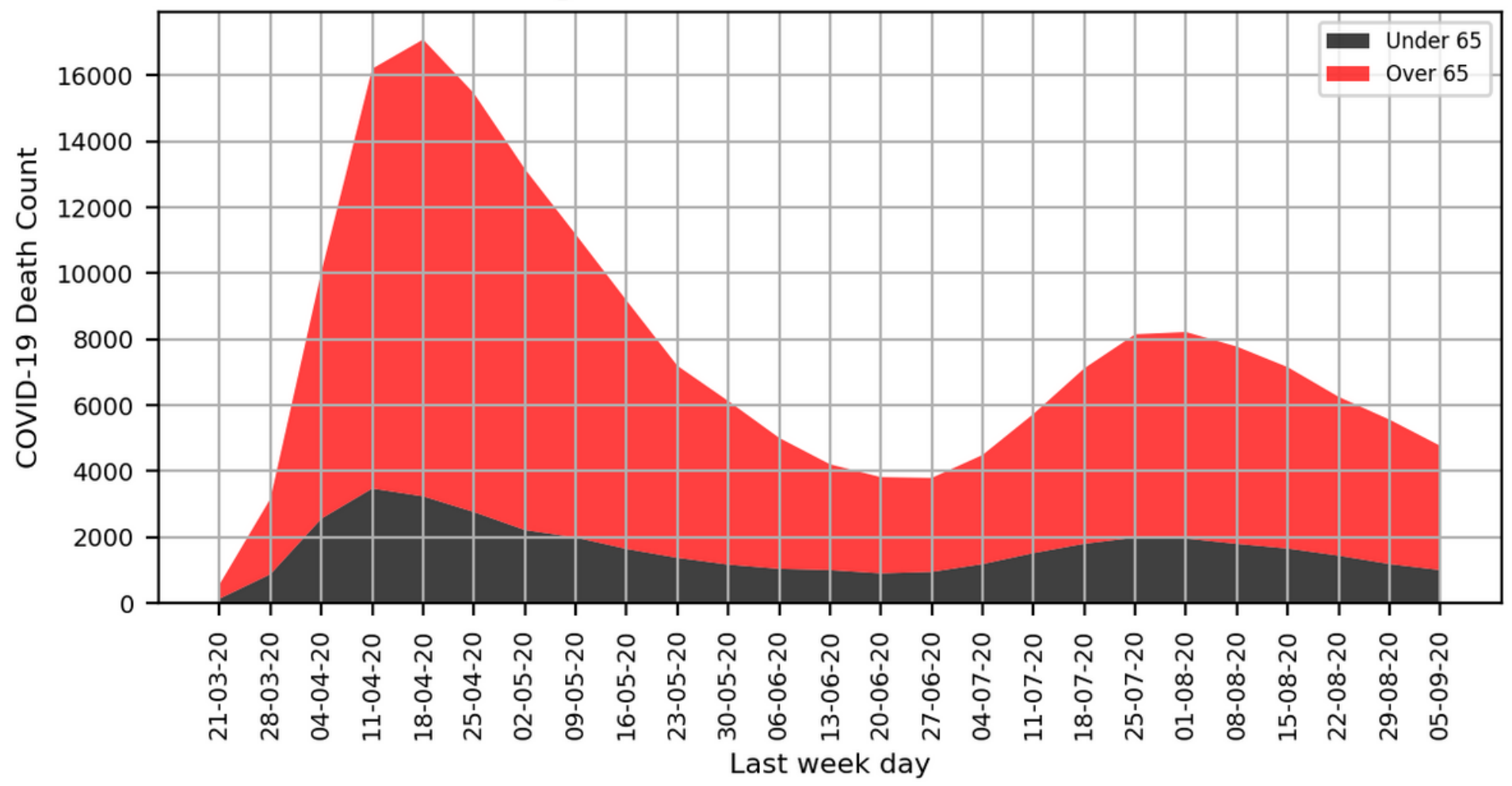

Figure 1

Weekly COVID-19 death count with age contribution. 


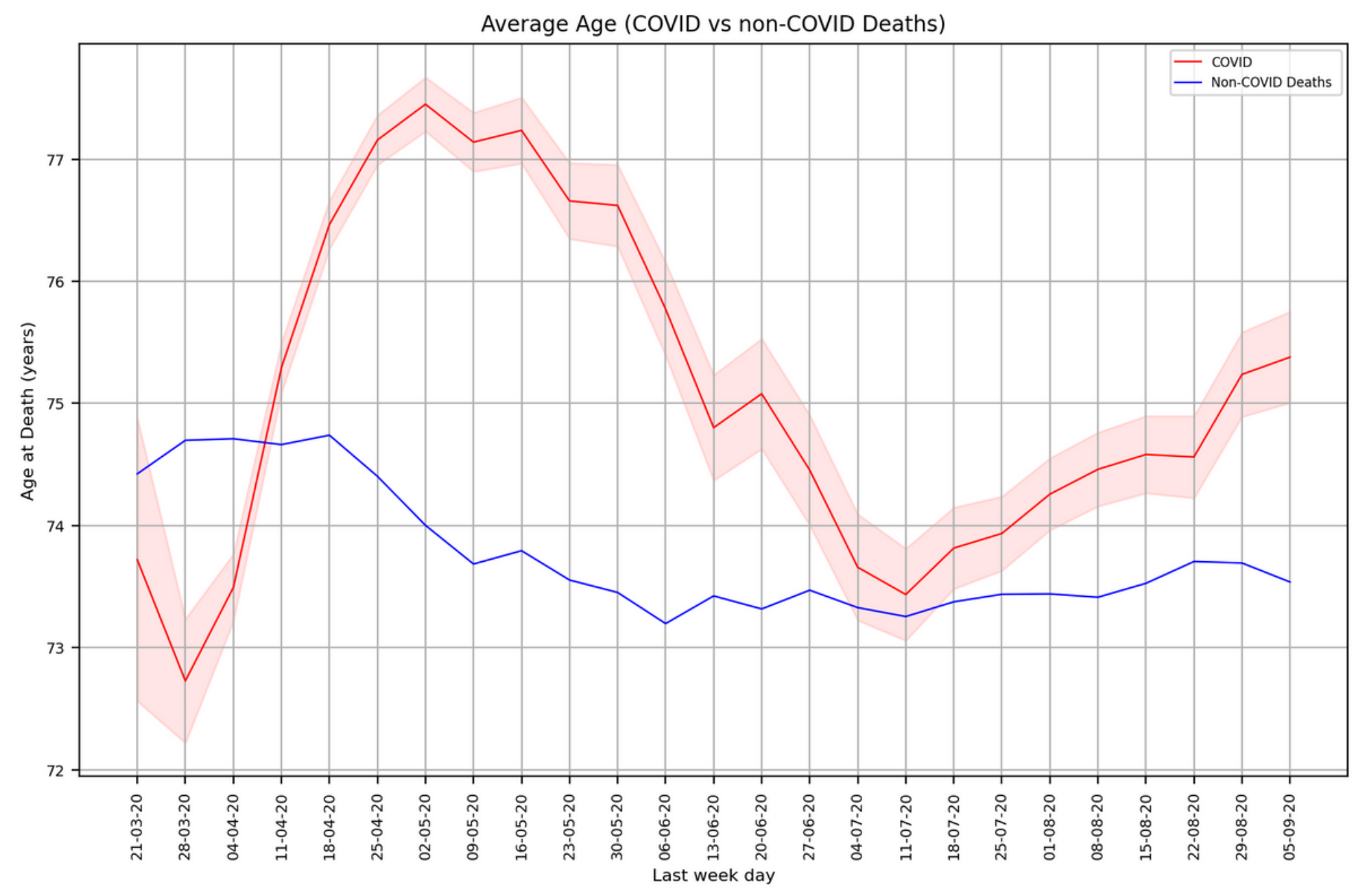

Figure 2

Ages at death for the COVID-19 and all other death categories (data is from National Center for Health Statistics CDC, 2020) 


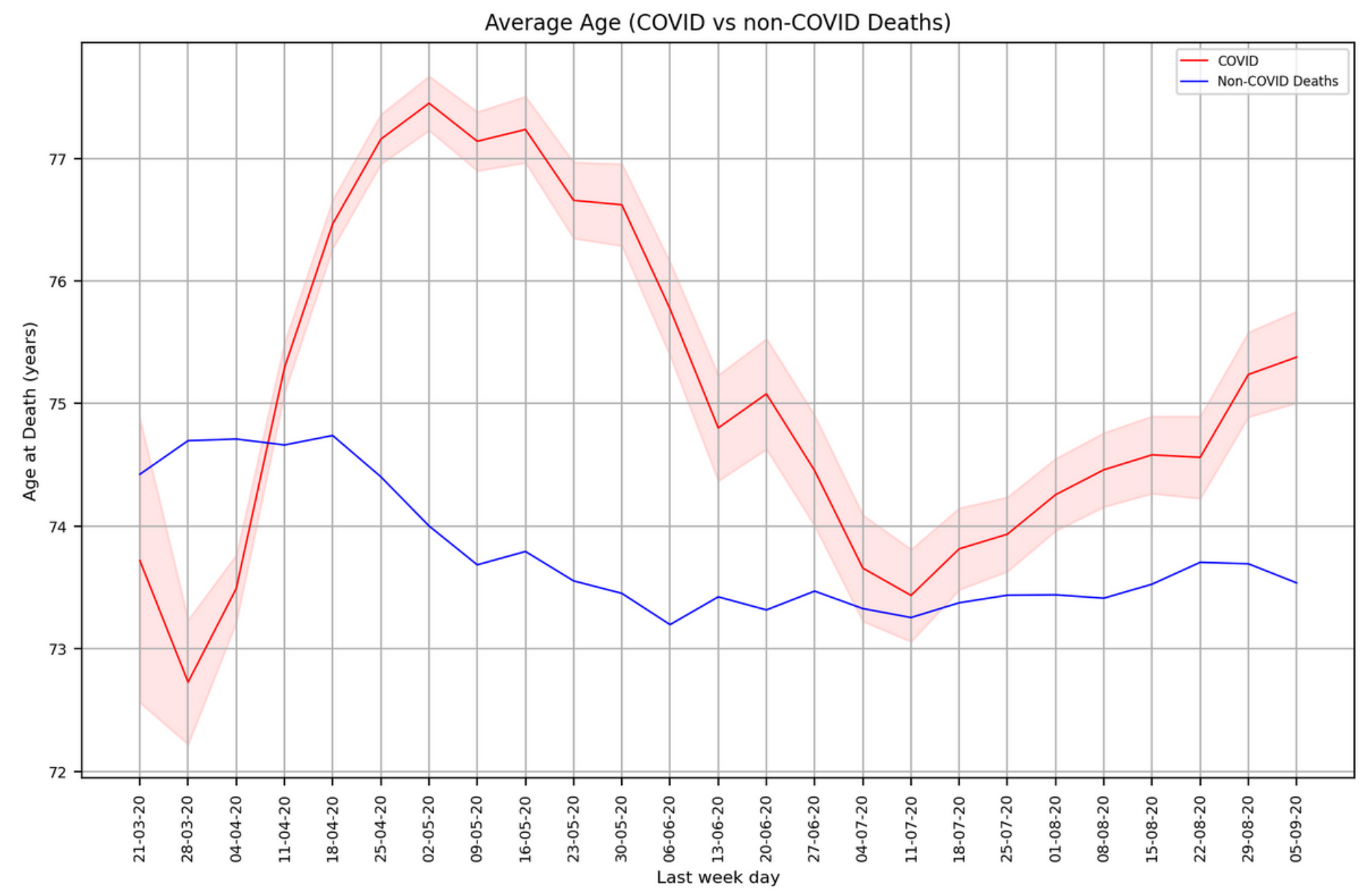

Figure 2

Ages at death for the COVID-19 and all other death categories (data is from National Center for Health Statistics CDC, 2020) 


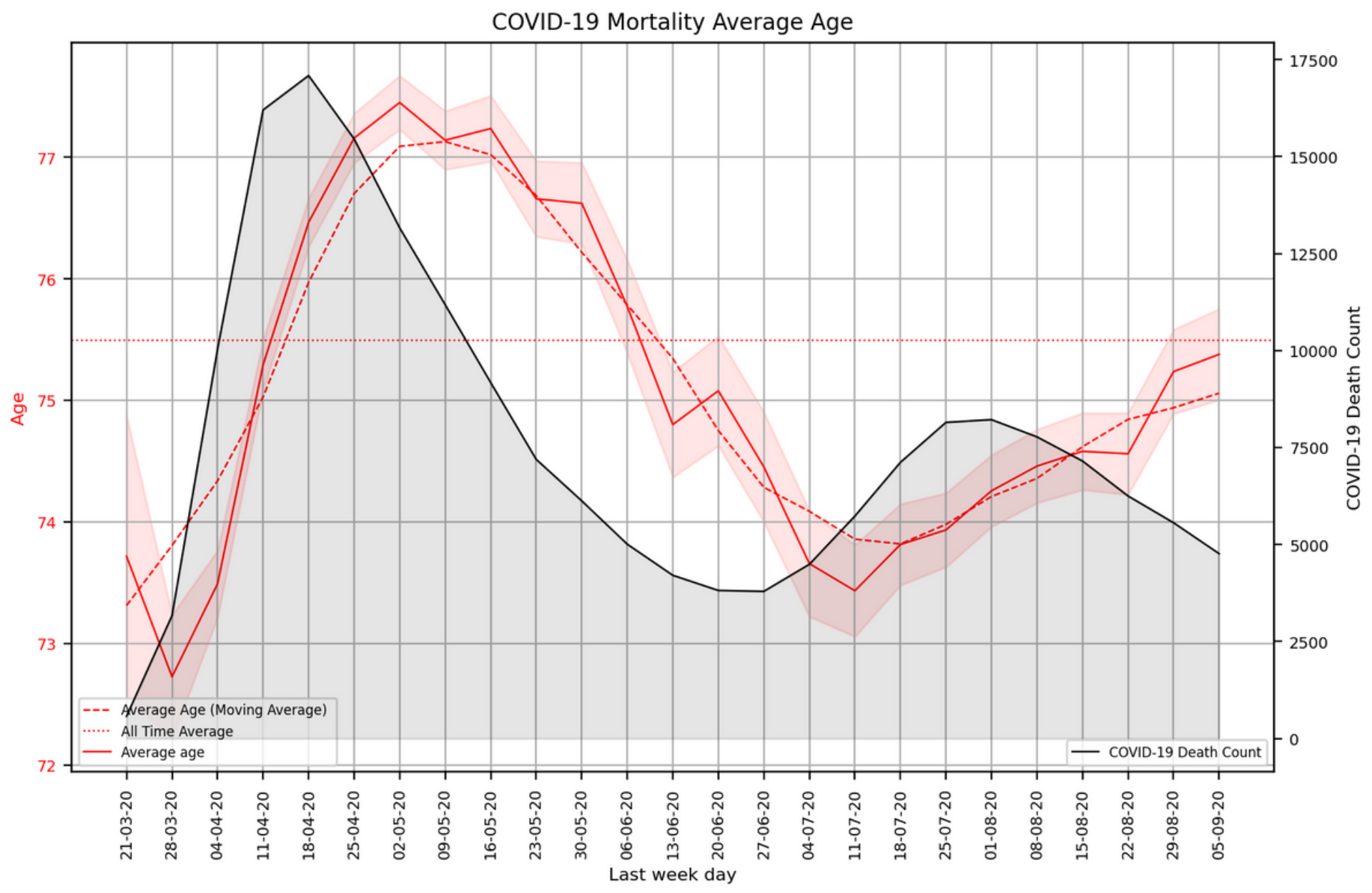

Figure 3

Weekly COVID-19 death counts in the US (in black) versus average age at death (in red), shown with the confidence intervals. 5-week moving averages are shown with the dashed and the overall weighted average with the dotted lines. 


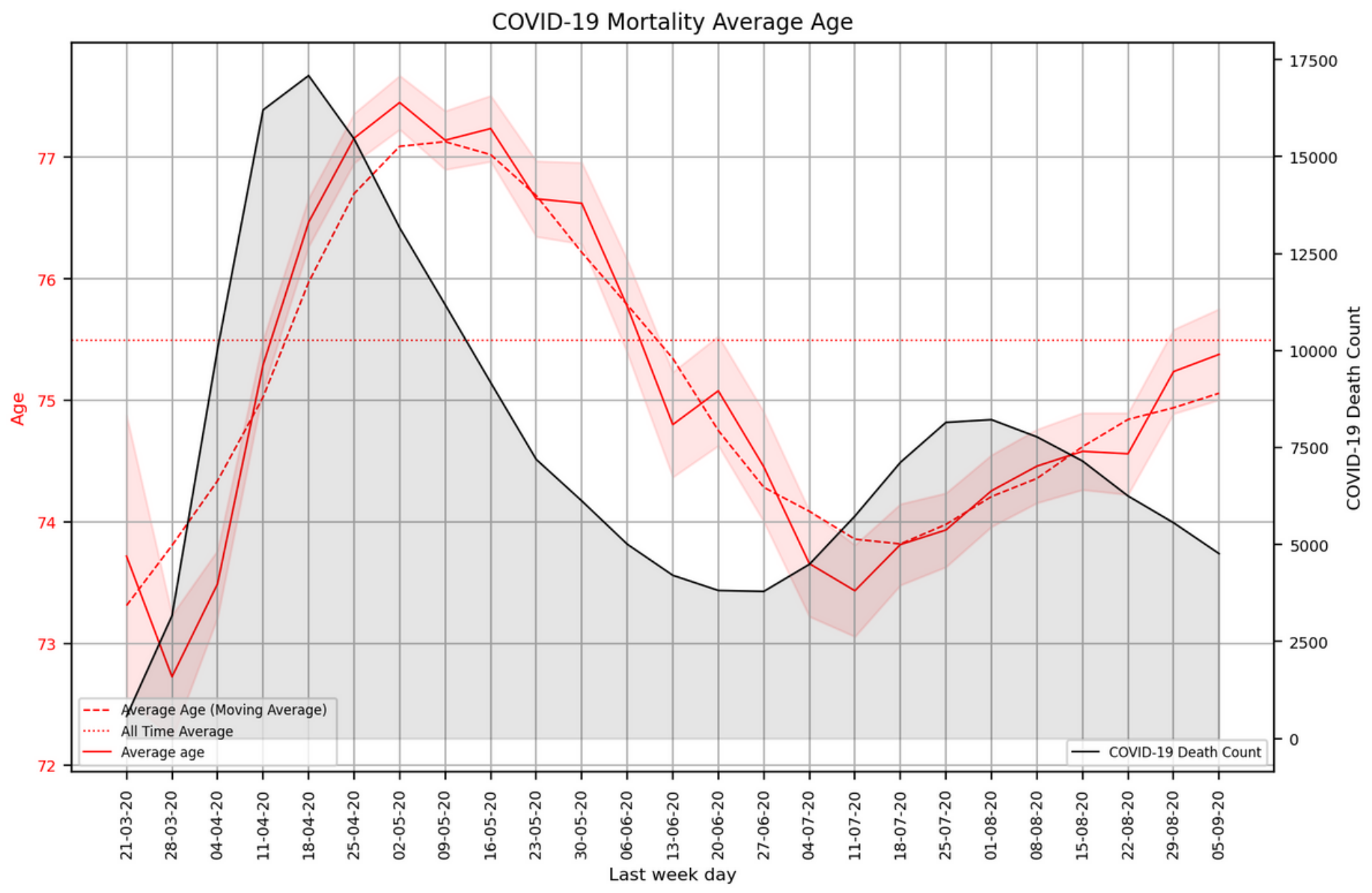

Figure 3

Weekly COVID-19 death counts in the US (in black) versus average age at death (in red), shown with the confidence intervals. 5-week moving averages are shown with the dashed and the overall weighted average with the dotted lines. 


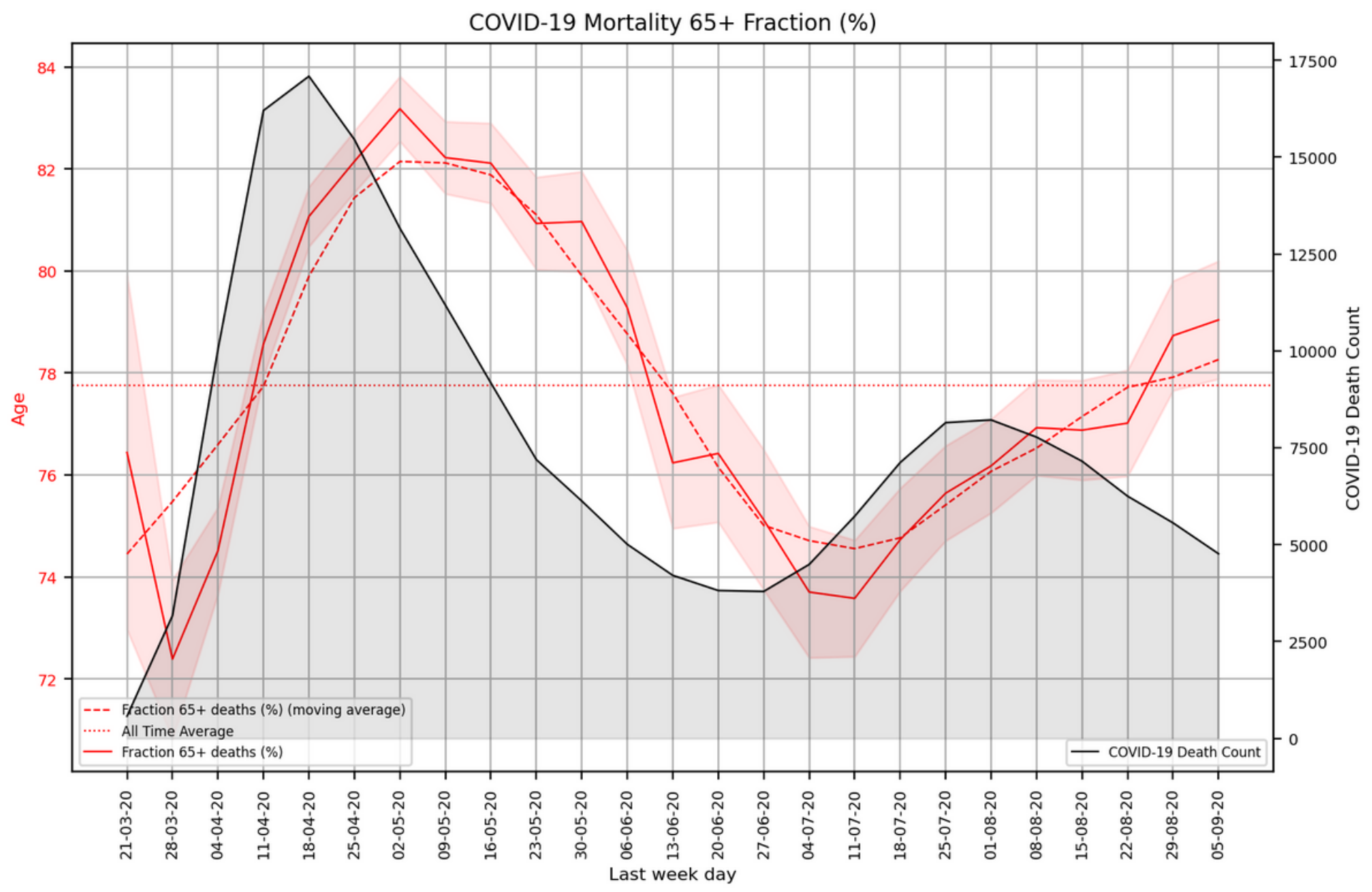

Figure 4

Weekly COVID-19 death counts in the US (in black) versus fractions of deaths at ages of 65 and over (in red), shown with the confidence intervals. 5-week moving averages are shown with the dashed and the overall average with the dotted lines. 


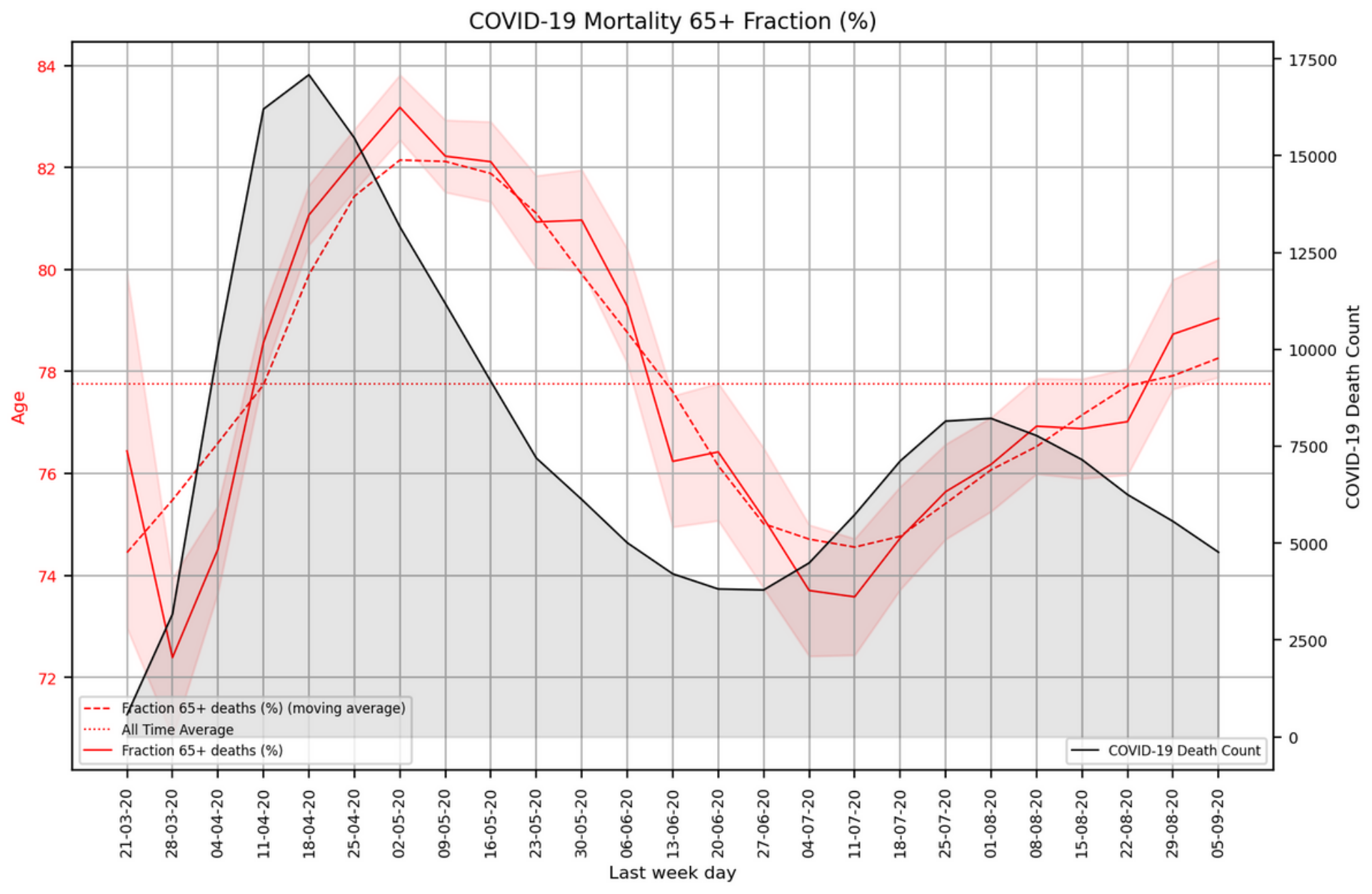

Figure 4

Weekly COVID-19 death counts in the US (in black) versus fractions of deaths at ages of 65 and over (in red), shown with the confidence intervals. 5-week moving averages are shown with the dashed and the overall average with the dotted lines.

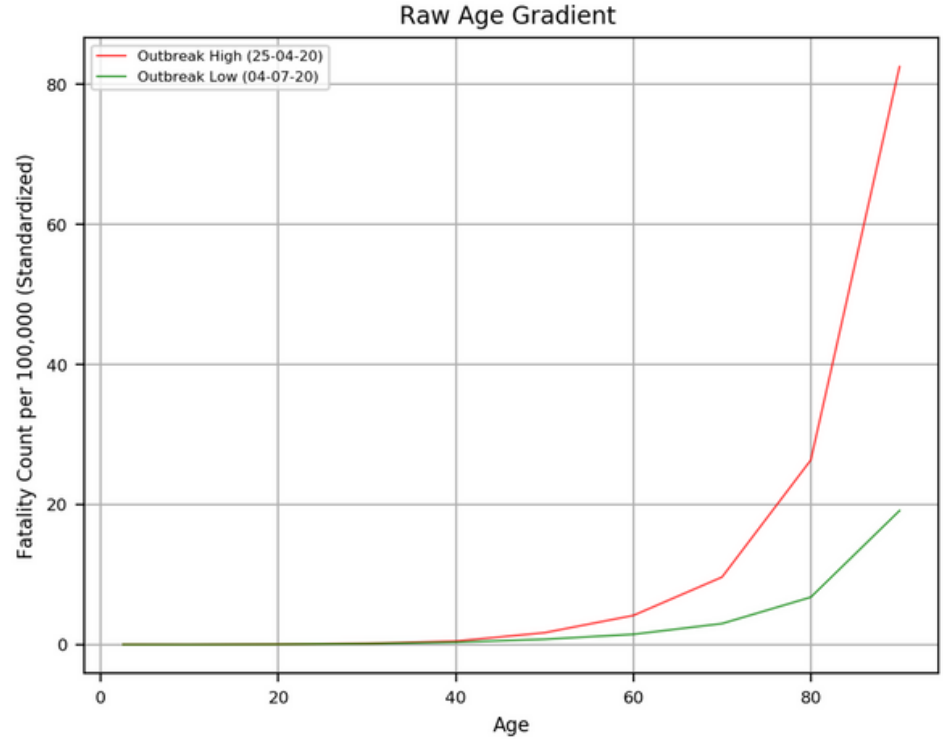

(a)

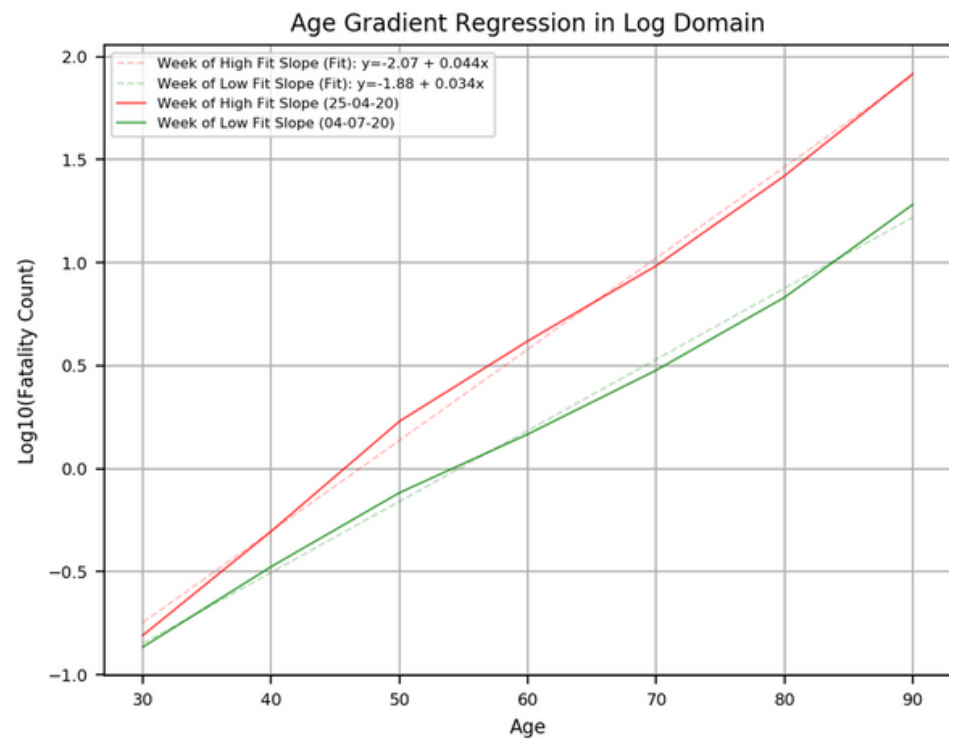

(b)

Figure 5

(a) Raw age gradient for high and low log regression slope coefficients for two select weeks; (b) Same in the logarithmic space with linear regression fit equations. 


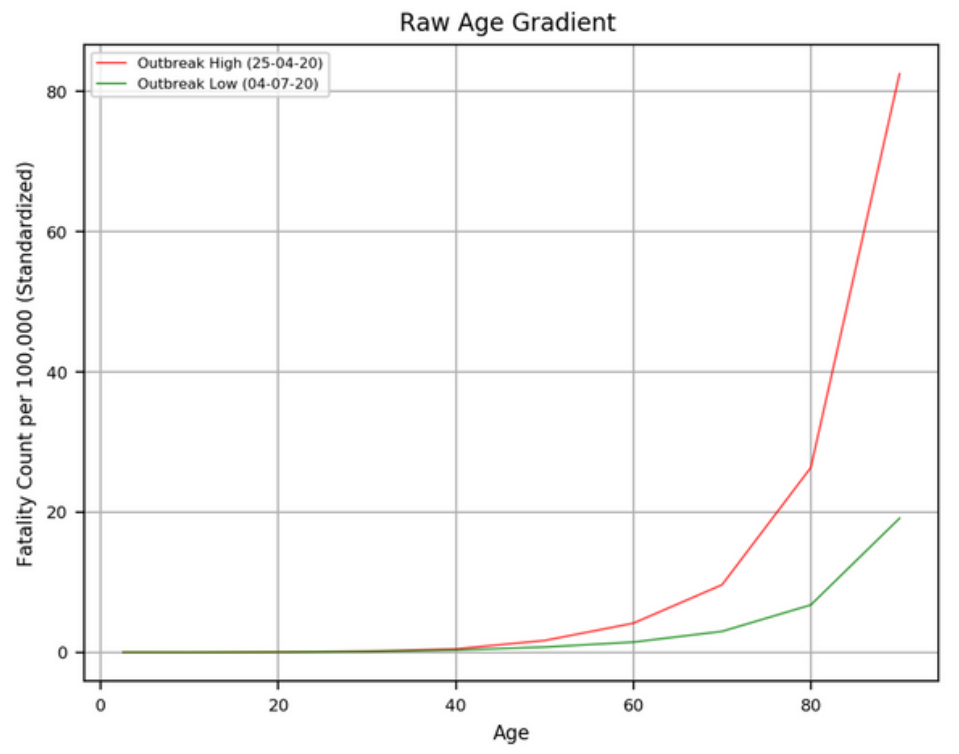

(a)

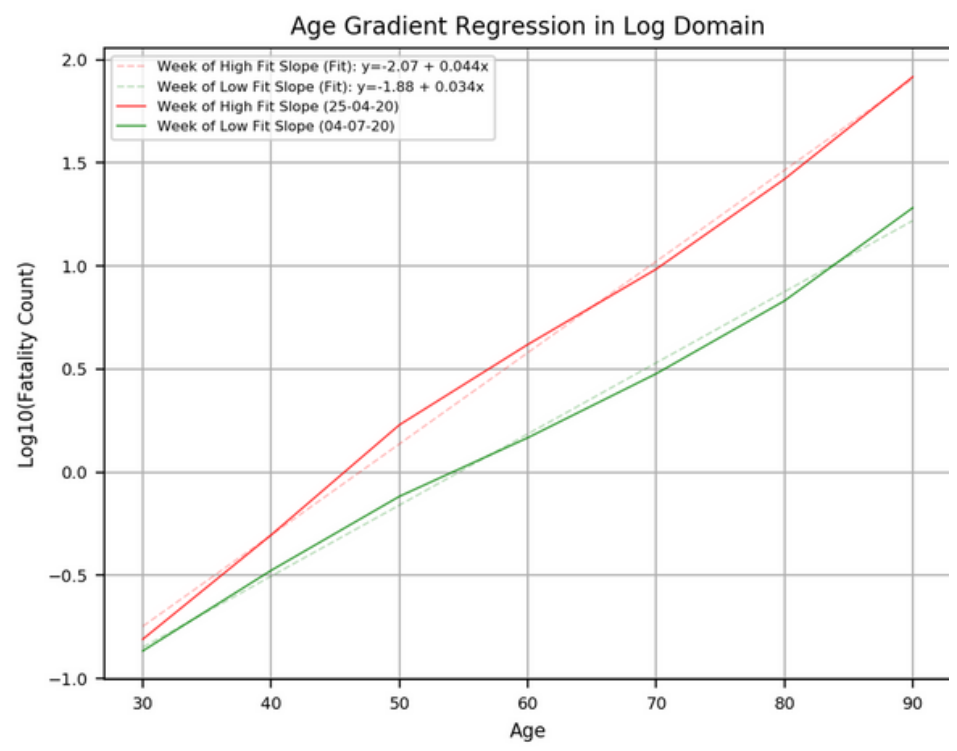

(b)

Figure 5

(a) Raw age gradient for high and low log regression slope coefficients for two select weeks; (b) Same in the logarithmic space with linear regression fit equations.

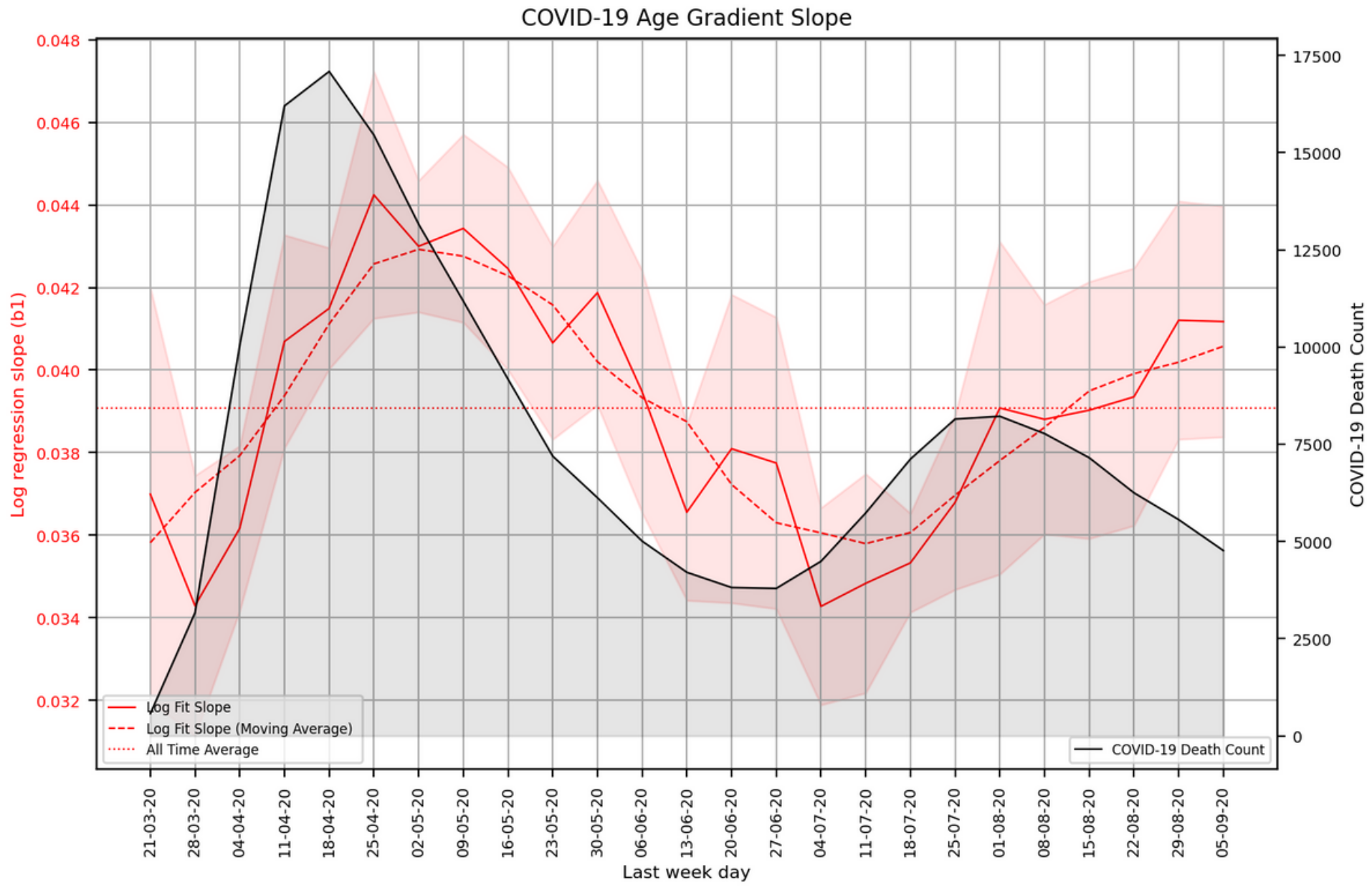

Figure 6

Weekly COVID-19 death counts in the US (in black) versus slope of linear regression fit of the mortality age gradients (in red), shown with the confidence intervals. 5-week moving averages are shown with the dashed and the overall average with the dotted lines. 


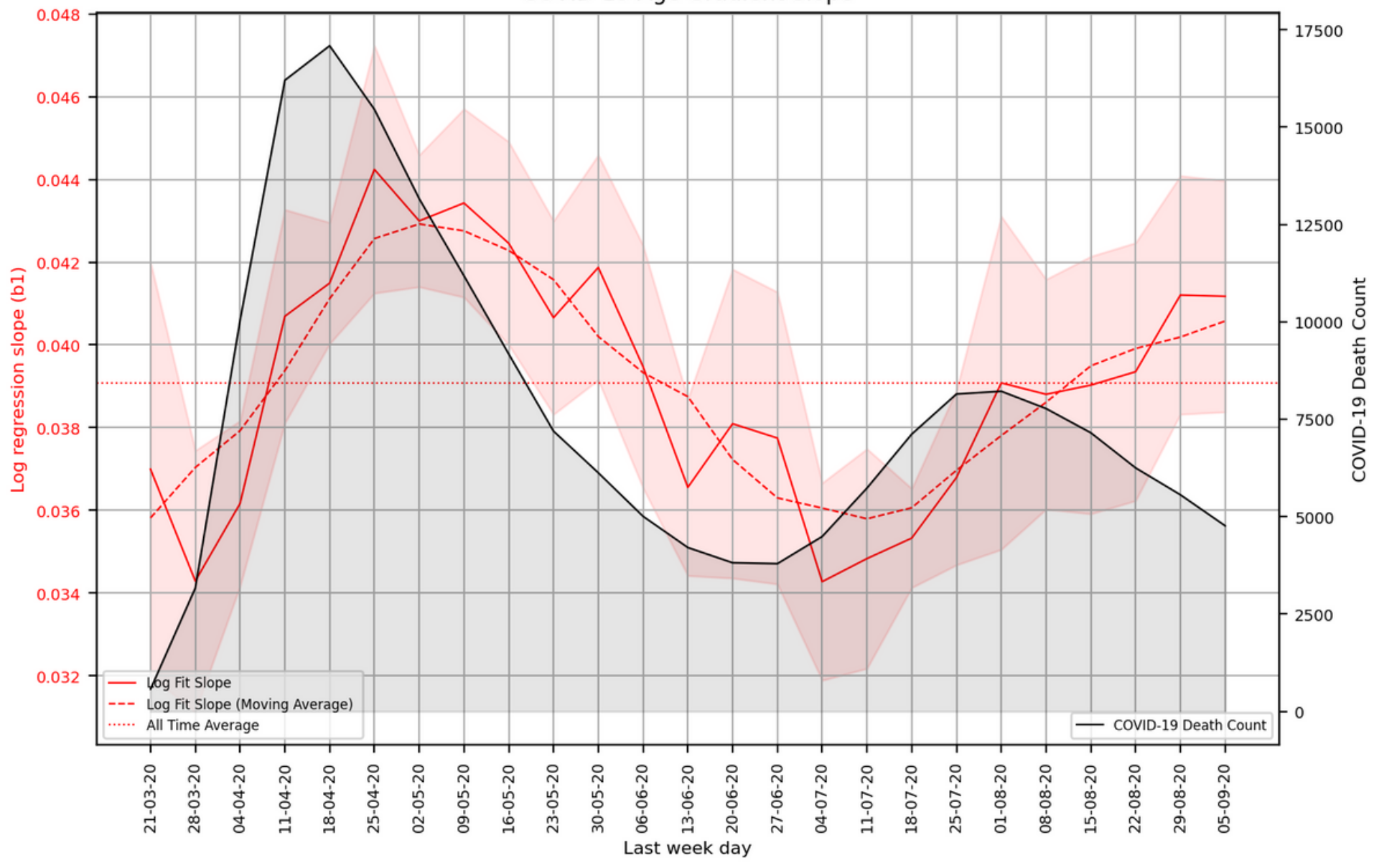

Figure 6

Weekly COVID-19 death counts in the US (in black) versus slope of linear regression fit of the mortality age gradients (in red), shown with the confidence intervals. 5-week moving averages are shown with the dashed and the overall average with the dotted lines. 